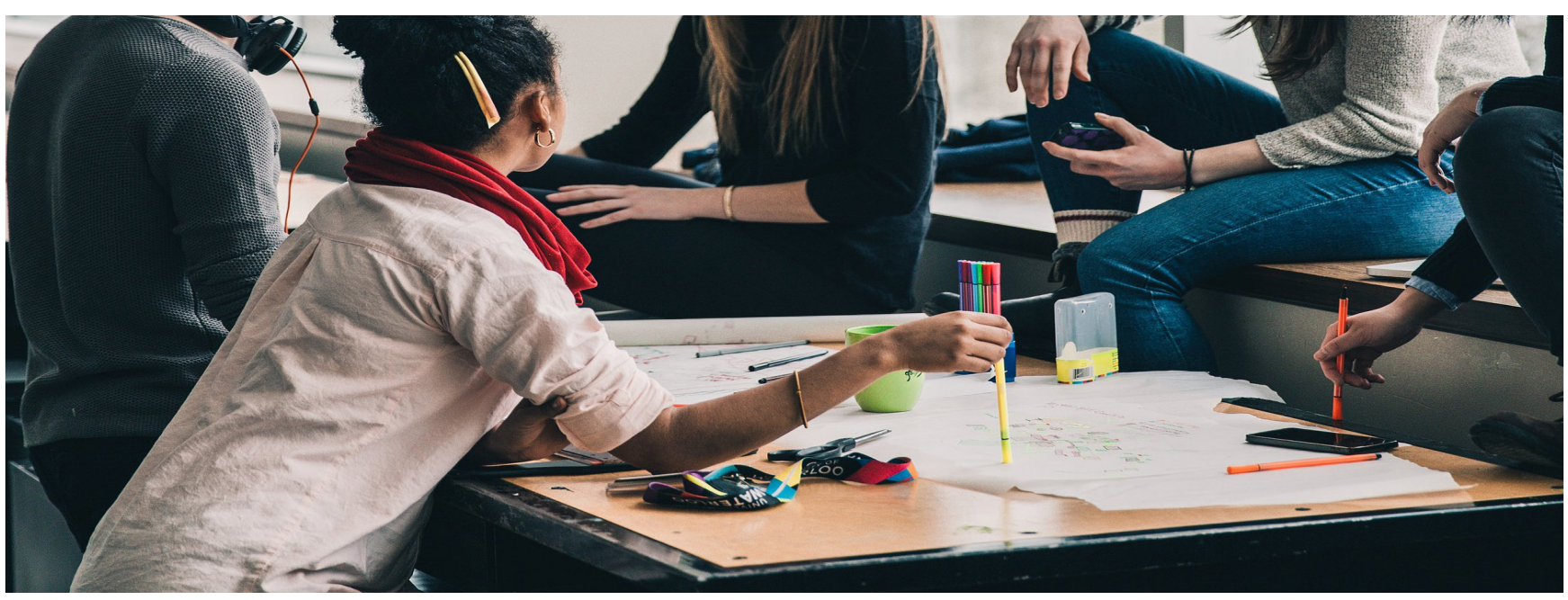

Tomado de: https://pixabay.com/es/personas-ni\%C3\%B1as-mujeres-estudiantes-2557396

\title{
¿Es posible la realización efectiva vía jurisdiccional de los derechos econó- micos, sociales y culturales?
}

\section{Is it possible the effective realization via court of economic, social and cultural rights?}

Gabriel Higuera-Licona ${ }^{1}$

\section{Resumen}

Los estados democráticos de derecho actuales encuentran el fundamento de su actuación en las ideas humanistas de los derechos humanos, que en su evolución persiguen incluso satisfacer las necesidades más apremiantes de la persona al menos, el mínimo vital. No obstante el camino de su realización efectiva, apenas comienza y no tiene una ruta fácil. No basta que se insista en ello, incluso por la vía jurisdiccional, si los derechos originales civiles y políticos no han alcanzado su pleno desarrollo. Si bien desde la parte progresista se estima que, se debe encontrar un contenido mínimo a proteger, que se deben de explorar modalidades creativas de la utilización de los recursos existentes, lo cual es muy razonable, pero no se debe dejar de lado las posturas realistas íntimamente relacionadas con el fenómeno económico.

Palabras claves: Estado democrático, derechos humanos, neoconstitucionalismo.

\begin{abstract}
The current democratic states with guaranteed rule of law find the basis of their action in the humanistic ideas of human rights, which in their progression seek to meet even the most important needs of the people or, at least, its vital minimum. Notwithstanding the road to its effective realization, this barely begins and does not have an easy route. It is not enough to insist on it even by judicial means, if the original civil and political rights have not reached its full development. As well, from the progressive party estimated that minimum content should find a protect, it should explore creative forms of the use of existing resources, which is very reasonable, but should not be left aside the realistic positions closely related to the economic phenomenon.
\end{abstract}

Keywords: Democratic State, human rights, neoconstitutionalism.

Fecha de recepción: 05 de noviembre de 2018/Fecha de aceptación: 12 de noviembre de 2018/*Correspondencia: gabrielhiguera1@hotmail.com/Universidad Autónoma de Tamaulipas, Facultad de Derecho y Ciencias Sociales Victoria/Dirección: Centro Universitario Victoria “Lic. Adolfo López Mateos”, Ciudad Victoria, Tamaulipas, México. C.P. 87149. 


\section{Introducción}

En el centro de las decisiones de Estado actuales, al menos de occidente, están inmersas en el tema de los derechos humanos, nuestro país dentro del influjo del concierto internacional de las naciones ha adoptado en la Constitución Política de los Estados Unidos Mexicanos, los principios rectores del neoconstitucionalismo, entre los que destacan el Control de Convencionalidad, el principio pro persona, y la interpretación conforme entre otros, trayendo cuando menos un nuevo paradigma en la interpretación de la Constitución, que si bien es fruto de los principios éticos más avanzados del pensamiento contemporáneo, encuentran algunas dificultades para su plena realización; lo que se observará en este breve ensayo, no desde el punto de vista jurídico ni mucho menos jurídico-filosófico, pues no es el objeto de este escrito, sino solamente observarlo desde un punto de vista meramente práctico o realista, pues debemos dejar muy claro que la teoría de los Derechos Fundamentales es lo mejor que le ha pasado a la relación entre gobernante y gobernado, lo que se ha traducido en las libertades públicas e igualdad ante la ley, trayendo consigo condiciones de progreso.

En el caso de nuestro país, a efecto de ser atendidos los principios del neoconstitucionalismo las fuerzas políticas tuvieron a bien a realizar en el año 2011 una reforma constitucional integral en materia de derechos humanos, que no obstante a su brevedad, cimbró el sistema jurídico, que de ser eminentemente legal puso a la Constitución en el centro del mismo sistema jurídico, convirtiéndola en directamente vinculante junto con los tratados y convenciones de derechos humanos de los que el Estado Mexicano forme parte.

Esto en realidad no es algo nuevo, toda vez que al menos desde el año 2007 el Poder Judicial de la Federación a través de sus diversos órganos jurisdiccionales invalidaban, por citar un ejemplo, sentencias del orden civil y familiar, porque los agentes del ministerio público, adscritos a los juzgados comunes desahogaban automáticamente las vistas sin manifestarse en una verdadera defensa; luego, estas resoluciones buscaban desde entonces alinearse con los tratados internacionales en la materia.

En este orden de ideas los Derechos Económicos, Sociales y Culturales en adelante DESC, son aquellos que tienen que ver con las condiciones del mínimo vital para una vida digna, como puede ser el acceso a una vivienda, la alimentación, al trabajo, a la salud, educación entre otros. De esta forma se realizará un breve análisis, en el cual se verán las dificultades para la plena realización vía jurisdiccional de los derechos enunciados anteriormente. Ya que el neoconstitucionalismo implica la aplicación de manera directa de los postulados de la Constitución por la judicatura, en consecuencia, a partir del inicio de la décima época de la jurisprudencia de la Suprema Corte de Justicia de la Nación, se ha reiterado que estos derechos pueden reclamarse a través de una tutela jurisdiccional efectiva.

\section{Diversas Interpretaciones del Poder Judicial, Respecto a los DESC}

El neoconstitucionalismo implica para una parte de la doctrina procesalista, solamente un cambio de paradigma de un legislador omnisciente a un juez omnisciente, de ahí que de acuerdo con la teoría del neoconstitucionalismo los DESC pueden reclamarse vía jurisdiccional, esto es, que a través de sentencias de los tribunales se consiga la plena realización de estos.

Dentro de esta corriente jurídica preponderante los Tribunales Constitucionales tienen mucho que decir, pues ahora en la interpretación directa de los principios y directrices que se desprenden de la Constitución y de los tratados en materia de derechos humanos, el derecho es a fin de cuentas los que los Tribunales Constitucionales acaban diciendo que es. 
Derivado de un análisis de los criterios emitidos por el poder judicial de la federación y de la Corte en particular a través de tesis y jurisprudencias, con especial énfasis en la décima época, podemos observar cómo se van configurando a la luz de la nueva forma de percibir el derecho en relación con los derechos humanos y en especial con la interpretación de los DESC, entre los que destacan los siguientes:

En primer lugar, que el Estado debe implementar las políticas públicas a fin de evitar que la persona se vea inconstitucionalmente reducida en su valor intrínseco como ser humano, "por no contar con las condiciones materiales que le permitan llevar una existencia digna” (Tesis I.4o.A.12 K, 2013: 1345)

En segundo lugar, que los Estados cumplen con la obligación de garantizar el núcleo esencial de los DESC "al establecer y fomentar las condiciones necesarias que permitan su ejercicio, sin que estén obligados a proporcionarlos de forma directa o gratuita, ya que se considera que las personas puedan satisfacer sus propias necesidades a través de su esfuerzo y trabajo". (Tesis $2 a$. XCIII/2016, 2016: 834)

En contraste con lo anterior, existen otros criterios jurisprudenciales en donde destacan que "el Estado debe tomar e implementar las medidas legislativas, administrativas, presupuestarias y judiciales adecuadas para la realización plena de dicho derecho”. (Tesis 1a. CXLVIII/2014, 2014: 801)

Por otra parte, ha dicho que la realización progresiva de, por ejemplo, el derecho a la salud a lo largo de un determinado periodo no priva de contenido significativo a las obligaciones de los estados, sino que les impone el deber concreto y constante de avanzar lo más expedita y eficazmente posible hacia su plena realización.

En ese mismo orden de ideas sigue diciendo que el Estado debe de demostrar que ha adoptado todas las medidas "hasta el máximo de los recursos" para lograr la realización de los derechos. (Tesis 2a. CIX/2014, 2014: 1190)

En general posturas jurídicas alineadas con la corriente de la realización efectiva de los DESC vía jurisdiccional, que se pueden resumir en que el Estado debe además de fomentar, adoptar todas las medidas para su materialización más eficaz.

Sin embargo, también se ha manifestado en un sentido, que si bien, no contradice totalmente las posturas antes señalas, si reconoce la dificultad de su realización efectiva en general, incluyendo entonces la vía jurisdiccional, cuando, por ejemplo, en la tesis aislada de la Primera Sala se menciona que "los principios de eficiencia y planificación que requieren que los órganos expertos y técnicos (se refiere a los estatales) sean los que conduzcan esos principios de política pública a una realización óptima, mediante la emisión de normas operativas que no podrían haberse previsto por el legislador". (Tesis 1a. CCCXVII/2014, 2014: 574). Es decir, reconoce implícitamente la dificultad de la aplicación efectiva de las políticas públicas para la administración, y desde mi óptica, con mayor razón para los jueces constitucionales. Máxime si partimos de la base que los órganos de la administración pública cuentan con estructuras técnicas especializadas, con las que los jueces no cuentan. De la misma manera el Poder Judicial ha determinado que en cuanto al alcance del artículo 1o constitucional "que si bien el verdadero sentido de este principio es colocar a los particulares en condición de poder acceder a los derechos protegidos constitucionalmente", y además que en relación al artículo 28 el cual prevé los principios de competencia y libre concurrencia, que el Estado está dotado de un conjunto de poderes que le permiten, vía regulación, incidir en la actividad económica de la 
población, con el objetivo de alcanzar los fines que el propio precepto enuncia. Reconociendo aquí el papel preponderante del Poder Ejecutivo en el desarrollo. (Tesis I.2o.A.E.32 A, 2016: 2740). Y por último, que en lo referente al alcance protector de los DESC "se espera que las personas puedan satisfacer sus propias necesidades a través de su esfuerzo y trabajo” (Tesis 2a. XCIII/2016, 2016: 834), esta postura en todo caso no requiere un desarrollo de los derechos. La Suprema Corte de Justicia de la Nación, actuando como un Tribunal Constitucional en la interpretación de los DESC, se observa mantiene por una parte una postura progresista que pugna porque el Estado siente las condiciones para la realización plena de estos Derechos, pero por otra parte existe reconocimiento de las dificultades técnicas y operativas que corresponde desarrollar a las diversas áreas que componen a la administración pública, a través de las políticas de desarrollo y planeación.

En este sentido, el máximo órgano jurisdiccional ha emitido criterios que consideramos reflejan esta difícil realidad, al ralentizar el avance de la corriente garantista, oportuna desde nuestro punto de vista, pues permite reflexionar con mayor cuidado las consecuencias de abrirla plenamente, así en una tesis polémica que se refleja en los votos concurrentes que se dieron, dejó asentado que "derivado de la parte final del primer párrafo del citado artículo 1o, cuando en la Constitución haya una restricción expresa al ejercicio de los derechos humanos, se deberá estar a lo que indica la norma constitucional, ya que el principio que le brinda supremacía comporta el encumbramiento de la Constitución como norma fundamental del orden jurídico mexicano, lo que a su vez implica que el resto de las normas jurídicas deben ser acordes con la misma, tanto en un sentido formal como material, circunstancia que no ha cambiado”.(Tesis P./J. 20/2014, 2014: 202).

\section{Diversas Opiniones Referentes a los DESC}

Es preciso señalar que la corriente progresista de los derechos humanos es la más influyente de nuestros días, pero no es doctrina absolutamente pacífica; solamente para efectos de carácter didáctico repasemos algunos influyentes autores sobre todo de América del norte, Estados Unidos de América y Canadá, en donde existe una corriente intelectual que considera que los DESC no son realmente derecho, a manera de ilustración John R. Searle uno de los filósofos más importantes de la actualidad, distingue entre derechos negativos (políticos y civiles) y los positivos que podríamos considerar los equivalentes a los DESC, señalando a este autor que existe una lista mínima de derechos humanos entre los que incluye el derecho a la vida, a la seguridad persona, a la propiedad, libre expresión y de asociación y que no deberíamos permitirnos hablar de derechos humanos a menos que se den los siguientes tres presupuestos:

1. Contra quién es el derecho;

2. Cuál es el contenido exacto de la obligación que se tiene con el portador del derecho; y

3. Exactamente por qué la persona contra quien existe el derecho está bajo esas obligaciones. (Searle, 2014:pp 252-253)

Citando a este autor, contempla que si, por ejemplo, el Estado de California en Estados Unidos decide que todos los ciudadanos de su entidad tengan garantizado el Derecho a una vivienda adecuada esto no es un caso de derechos humanos sino de derechos de los ciudadanos de un Estado en particular promulgados por el poder legislativo de dicho Estado (2014), es decir considero que se refiere este autor a las decisiones de Estado en cuanto a las políticas públicas que ha de implementar.

Por su parte la también controvertida filósofa Ayn Rand considera, en similar sentido que en tratándose de lo que ahora consideraríamos los DESC, hace dos preguntas iA costa de quién? y ¿Quién debe proporcionarlos?, sigue señalando que el derecho de un hombre no incluye la materialización de ese derecho por parte de otros hombres, en todo caso que los padres fundado- 
res de los Estados Unidos hablaban del derecho a la búsqueda de la felicidad no del derecho a la felicidad. Esto significa que un hombre tiene el derecho a realizar las acciones que considere necesarias para la lograr su felicidad, pero no que otros deban hacerlo feliz. (1964, pp 139-140). Lo anterior manifiesta que ambos autores coinciden en el sentido de que si bien las constituciones de los Estados están enfocadas en buscar el mayor estado de bienestar, corresponde a cada uno de los ciudadanos la búsqueda y realización efectiva de los mismos.

El jurista de Harvard David Kennedy (2007), expone que la posición de los derechos humanos como proyecto político emancipador que se extiende y opera en un ámbito más allá o por encima de la política, (un proyecto político vuelto a empaquetar en forma de conocimiento) deslegitimiza otras voces políticas y hace menos visibles las dimensiones locales, culturales, y políticas del propio movimiento de derechos humanos. (pp 81-82).

Por su parte el político canadiense Michael Ignatieff, señala que algunos derechos humanos que podríamos identificar con los DESC, por ejemplo, la autodeterminación de los pueblos, la vivienda digna, una adecuada alimentación, el agua, etc; Entendidos como derechos humanos degradan el objetivo mayor de estos, es decir, de la "agencia humana" que este autor entiende como la capacidad que posee un individuo para lograr sus deseos racionales sin obstáculos ni impedimentos. (Arias y Sanchez. 2008, pp 187-201).

Estos autores en general coinciden en que, en dado caso, hay un derecho a la búsqueda de los satisfactores por nuestro propio impulso, pero no a que nos sean proporcionados por otros. y que la corriente preponderante de los derechos humanos impide que se materialice o que se ejerza plenamente la acción política, podremos o no coincidir con estos autores, sin embargo, los razonamientos no dejan de tener argumentos considerables.

En las antípodas de las posturas antes relatadas se encuentra por otro lado, la corriente pro-derechos humanos, en este caso me concretaré a citar a algunos autores que razonan bien la defensa incluso jurisdiccional de los DESC, Ligia Bolivar (1996) y Abramovich y Courtis (2002), estos últimos ya citados, se decantan por una realización efectiva, incluso jurisdiccional. La primera de las mencionadas señala de forma inteligente, que es un mito la diferencia entre los derechos civiles y políticos con los DESC, en cuanto que hay derechos clasificados en el grupo de civiles y políticos que requieren del Estado una asignación de recursos suficientes como el caso del derecho del detenido, en el que no se puede argumentar un retraso en la excarcelación por falta de recursos económicos del Estado. Esta afirmación al parecer contundente, si la analizamos con mayor detalle, nos damos cuenta que en realidad debe de matizarse. El Estado debe su existencia de manera preponderante para establecer la paz y tranquilidad de sus habitantes y en esta tarea sienta las bases jurídicas y de infraestructura para sancionar las conductas de quien viole la ley, que si el Estado es funcional, son una minoría de sus integrantes quienes transgreden la norma, en el caso de los DESC, dirigidos a toda la población, encuentra una barrera natural en el tema de recursos monetarios que el Estado debe destinar, en sentido ideal a toda la población.

Los autores antes mencionados que defienden la exigibilidad de los DESC incluso vía jurisdiccional coinciden en que se deben encontrar un contenido mínimo a proteger, que se debe de explorar modalidades creativas de la utilización de los recursos existentes, superando los mitos de la distinción entre los derechos civiles y políticos y los DESC, y una creación de indicadores que en lugar de que estén diseñados en función de evaluar la ejecución de políticas, evalúen los resultados en materia de derechos humanos. 
Si bien pudiera ser una medida justa la creación de un contenido mínimo al que todo el mundo tuviera acceso, es importante establecer que encontrar este equilibro común es un proceso complicado ya que entran en juego factores externos difíciles de controlar que más adelante abundaremos, tomando como referencia al derecho a la educación.

Es cierto que, como hace notar esta corriente de pensamiento progresista que hay DESC que se pueden dirimir en los tribunales, sobre todo aquellos que tiene que ver con los menores, la familia, libertad sindical, protección de la cultura, trabajo, protección de minorías entre otros, que si bien son decisiones que inciden incluso a veces de manera trascendente en la políticas públicas, los que están en estrecha relación con la capacidad, económica del Estado, se insiste, se deben considerar progresivos en relación directa con la capacidad económica del Estado, partiendo de la base de lo que este puede invertir en servicios e infraestructura es lo que recauda, complementado, en todo caso, con deuda pública que de manera responsable puede adquirir. Es decir, lo que la teoría en pro de la realización efectiva vía jurisdiccional de los DESC no explica, es como enfrentar la realidad económica de un Estado con la noble aspiración a que todos tengamos los satisfactores suficientes; no basta decir que se deben buscar soluciones creativas. Partiendo de la premisa que ante un determinado presupuesto limitado, lo que se invierta en un servicio específico se estará dejando de invertir en otro. El impulso ético de esta corriente es innegable y deseable, desde luego, pero choca de manera ineludible con el aspecto económico, ya que como todos sabemos el fin último del Estado es velar por el bien común, mismo que solo puede satisfacerse con las asignaciones presupuestarias que el Estado determine implementar derivado de la tasa recaudatoria, es decir, difícilmente el gobierno podrá solucionar los problemas de manera particular cuando la recaudación es poca, por lo que es una labor necesaria buscar el correcto equilibro de las necesidades de la población en lo general, buscando el beneficio colectivo antes que el individual.

\section{Dificultades de su Implementación}

Por otra parte, estamos de acuerdo con Abramovich y Courtis (2002) cuando señala que es importante la protección jurisdiccional de los Derechos humanos en tratándose de la protección al derecho a la salud y medio ambiente de las comunidades originarias, o de atender a las necesidades de los enfermos de VIH. Sin embargo, estos mismos autores reconocen insuficiencias tratándose de los que tienen que ver con acciones positivas del Estado, es decir obligaciones de dar, como es el caso de los derechos a la alimentación, vivienda, salud etc; pues aquí nos encontramos con dificultades prácticas o realistas sino insalvables sí muy difíciles de superar, realistas partiendo de la base de la utilidad de la norma jurídica en relación con la realidad, como señala Enrique P. Haba (2002) "una percepción de la falta de correspondencia entre el carácter abstracto de la normas generales y la individualidad de los casos concretos", en el entendido que lo mejor sería la correspondencia de la norma y la realidad social. Entonces, pongamos por caso, el derecho a una vivienda digna y sólo para fines ejemplificativos vayamos al extremo que 10 millones de personas (si consideramos los márgenes de pobreza de CONEVAL que en el año 2014 rondo los 46 millones de pobres en nuestro país) promovieran un juicio de amparo al tenor del contenido del artículo 40 de la Constitución, por considerar que no tienen una casa con condiciones adecuadas de habitabilidad. Cabrían dos escenarios en primer lugar, que los jueces federales resolvieran, en congruencia con criterios anteriores en cuanto que el Estado debe proveer las condiciones necesarias, creando una estrategia nacional de vivienda, asegurando las medidas legislativas, administrativas, presupuestarias y judiciales para la realización plena de dicho derecho. Criterios que se reflejarían poco en la realidad vital de los gobernados y reforzarían la idea que son derechos programáticos que su reconocimiento pleno es progresivo, de conformidad con las condiciones económicas estatales. 
En el otro hipotético escenario, amén de las complicaciones numéricas que esto traería, (con el seguro quebranto de la operación administrativa del Poder Judicial de la Federación), que los jueces federales decidieran conceder la protección de la justicia federal, a fin de que se construyeran las viviendas con las condiciones de construcción adecuadas y suficientes, obligaría a la Administración Pública a dejar de invertir en ciertas áreas, incluso prioritarias, para cumplir con las sentencias, lo que, por lo menos, es un nuevo paradigma en las funciones tradicionales del gobierno, en relación con la separación tradicional del poder, es decir, se corre el riesgo de un activismo judicial cuando menos.

Comparto la idea de la importancia de la realización efectiva de los DESC incluso desde la importante trinchera de la defensa vía jurisdiccional, pero se aprecia su insuficiencia que considero debe ser complementada con la acción política de la Administración Pública.

A fin de cuentas, cualquier Estado moderno democrático aspira a que su sociedad goce de los satisfactores necesarios; desde Santo Tomas de Aquino, que consideró el bien común como la unión entre gobernante y sociedad, y siendo el primero el conducto para tal fin; pongamos por caso, en relación al derecho a la vivienda, el Estado mexicano cuenta desde los años setenta del siglo pasado con un instituto de fomento a la vivienda de los trabajadores, mucho antes de que la idea de los DESC incluso tuviera cierta influencia internacional. Debemos de reconocer, aunque como ya vimos la corriente preponderante que considera la realización efectiva vía jurisdiccional equipara a los Derechos civiles y políticos con los DESC, dicho razonamiento debe ponderarse en relación con factores de índole técnico y económico.

Por otra parte, para poder darnos cuenta de la complejidad de las decisiones que desde su realización efectiva vía jurisdiccional, tienen que tomar los jueces, basta citar como ejemplo, bajo qué parámetro puede un juez de amparo, pongamos por caso, decidir si el Estado mexicano ha propugnado por un derecho "hasta el máximo de los recursos" si refiriéndonos al derecho a la educación, por ejemplo, estamos hablando de un presupuesto asignado para el año 2017 a este rubro de $\$ 267,655,185,221.00$ y a su vez que en el año 2016 fue de $\$ 302,986,555,681.00$, presentándose una reducción de 35 mil millones de pesos sin duda, fruto de las fluctuaciones de la economía internacional, o bien, que México invierte el $81 \%$ del gasto total de educación en la remuneración de los docentes, o en educación superior un 62\%, cuando en los países de la OCDE (2016) el promedio es de $62 \%$ y $40 \%$ respectivamente; o que ha incrementado su gasto del PIB en educación del $4.4 \%$ al 5.2\%, ¿esto es poco o suficiente? para tomar una decisión en ese sentido tendría que ser experto en desarrollo, planeación, en general en políticas públicas y tener a su disposición en todo caso un cuerpo de asesores como se tiene en las secretarías de Estado del Ejecutivo Federal, con el agravante además que en el juego de la separación de poderes, el Ejecutivo federal y los estatales están sujetos al ejercicio del presupuesto; es decir, la distribución del gasto debe realizarse sujetándose a las disposiciones legales aplicables realizando las etapas de evaluación y planeación, además de que según lo dispuesto en el artículo 8 de la Ley Disciplina Financiera de las Entidades Federativas y los Municipios, mismo que dispone que "No procederá pago alguno que no esté comprendido en el Presupuesto de Egresos, determinado por ley posterior o con cargo a Ingresos excedentes", esto significa que los encargados de la administración de la política pública no pueden realizar erogación alguna sin estar debidamente establecida en el presupuesto de egresos correspondiente.

Esto, a propósito de la Tesis de la Primera Sala de la Suprema Corte de Justicia de la Nación, que en tratándose de la posibilidad para la autoridad de adoptar medidas regresivas en materia de derechos humanos, deja abierta la puerta siempre y cuando: "a) Se acredita la falta de 
recurso, b) Se demuestre que se realizaron todos los esfuerzos necesarios para obtenerlos, sin éxito; y c) Se demuestra que se aplicó el máximo de los recursos o que los recursos de que se disponía se aplicaron a tutelar otro derecho humano y no cualquier objetivo social", (Tesis 1a./J.87/2017 10 época), imponiéndole a cualquier servidor público una carga que en dado caso solamente a los titulares de una Secretaría podrían decidir. De las que no se puede hacer responsable, ni mucho menos cumplir, por no corresponder en muchos casos a su ámbito de decisión.

Abundando sobre las dificultades de carácter práctico o realista, me parece que, amén de considerar a diversos procedimientos administrativos y jurisdiccionales como parte del bloque de defensa jurisdiccional de los DESC, es la función de garantes por antonomasia de la Constitución y los derechos humanos que desarrollan los Tribunales del Poder judicial de la Federación vía el juicio de amparo, a quienes de manera preponderante corresponde la protección jurisdiccional de los DESC, pero la resolución de los asuntos sometidos a su jurisdicción caso por caso, propio de la técnica del juicio de amparo, aparte de lo poco significativa numéricamente hablando que sería la protección otorgada, (con excepción de aquellas resoluciones que generaran efectos generales a través de las declaratorias generales de inconstitucionalidad), considero que también sería injusta, partiendoprecisamente que por medio del juicio constitucional sólo se ampara a quién lo promueve, entonces, ante un mismo supuesto sólo obtendría la protección de sus derechos quién considerara estar en esa desventaja, situación de por sí muy subjetiva, o peor aún, quien tenga los recursos económicos para sufragar el juicio, en franca contradicción con la doctrina humanista propia de la defensa de estos derechos.

Ahora bien, es posible aspirar a la realización plena, cuando no estamos como sociedad dispuestos a cumplir la parte del pacto que nos corresponde; me explico, lo que un Estado nacional puede invertir en beneficio de la población está directamente relacionado, como ya comentamos, con la captación de recursos vía fiscal que recaude, y solamente eso. Como señala Casar, M. (2015) "México está en el peor de los mundos posibles. Clasifica entre los países de menor recaudación, menos del 10\%, y entre los de más alta evasión, entre 30\% y 40\%”. (p. 42) Señala esta misma investigadora, que esto se explica por la creciente desconfianza de la ciudadanía en la capacidad del gobierno para proveer servicios con el dinero que recauda, y que también es cierto que desgraciadamente hay muestras evidentes de que esto es así, basta saber que este mal endémico le cuesta a nuestro país 347 mil millones de pesos.

Sin embargo, será desde la misma acción política que tendrá, si queremos subsistir como Estado-democrático, resolverse y poner remedio a esta grave situación de corrupción que nos desgarra como nación. Debemos reconocer que existe un descrédito de las acciones de gobierno, de las que se tienen una percepción bien ganada de corrupción y de ineficacia, que no satisfacen las expectativas de los gobernados, en los aspectos que más interesan, el económico, social, de justicia, de paz, de desarrollo; sin embargo, se tiene que insistir desde la misma acción política para corregir esas graves deficiencias de la administración. Una acción política fundada precisamente en los derechos humanos, en aquellos políticos y civiles que sientan las bases de la igualdad, de la libertad, de la democracia y del desarrollo, pues si bien existe la tendencia a dar mayor importancia los derechos de nuevo cuño, se debe considerar primero la plena aplicación y vigencia los derechos políticos y civiles y que sean el piso mínimo para la plena aplicación DESC.

Aunque la doctrina más avanzada de los derechos humanos ya desde hace algún tiempo y actualmente con mayor ahínco pugna por la defensa efectiva de estos incluso de los DESC, ya desde principios de este siglo Víctor Manuel Rojas Amandi señalaba "los derechos humanos de carácter social y cultural tienden a fijar las bases materiales que posibilitan la existencia de los de- 
rechos humanos tradicionales", y añade "Una sociedad donde el analfabetismo sea mínimo donde exista un compromiso ciudadano hacia las actividades públicas, donde la distribución del ingreso sea equitativa, es la condición necesaria para la existencia de los derechos humanos”. (2009: 206)

Consideramos sin embargo que contrario a la opinión de este autor es evidente que el progreso social que menciona, solo se puede lograr donde los derechos y libertades fundamentales de primera generación, es decir los políticos y civiles son una realidad, donde la soberanía del pueblo se traduce en una democracia, donde el Estado de Derecho sea una realidad, en el que la seguridad jurídica y la seguridad ciudadana, la paz y la tranquilidad sienten las bases del desarrollo. Más bien coincidimos con Martín Diego Farrell (199 en cuanto que "los derechos políticos básicos habilitan a los titulares de derechos constitucionales a afirmar su pretensión sobre los recursos futuros, influenciando la política pública que hará posible la implementación completa de los derechos humanos correspondientes" (1997:130-138)

Desde luego, no puede dejar de insistirse en la realización plena de los derechos de última generación ¿Qué sociedad integrada no quisiera que esto fuera una realidad? Pero los hechos se imponen y diversos factores políticos y económicos se convierten en determinantes para su aplicación integral.

\section{Conclusiones}

Es tal la influencia actual, en las políticas públicas de los derechos humanos que muchas voces en nuestro país insisten en su realización plena, incluso vía jurisdiccional; si bien es lo deseable por la influjo ético y humanista que estos conllevan, las condiciones económicas lo impiden aun llevando a cabo las estrategias de los defensores de esta corriente que se debe reconocer han conseguido resultados significativos en algunas áreas, pero es pronto para considerar su efectividad completa. Es necesario consideramos, primero la vigencia de las políticas públicas que tengan su fundamento en los derechos humanos de primera generación, y luego, conseguir su vigencia más plena, ojalá en un futuro cercano se logre por el bien todos.

\section{Referencias}

Abramovich, V. y Courtis C. (2002). Los derechos sociales como derechos exigibles. Madrid: Trotta.

Farrell, M. (1997). Utilitarismo, liberalismo y democracia. México: Fontamara. 130-138 Pp.

Kennedy, D. (2007) El lado oscuro de la virtud, trad. de Francisco J. Contreras e Ignacio de la Rasilla, España: Almuzara. 81-82 Pp.

Rand, A. (1964). La virtud del Egoísmo, trad. de Luis Kofman, Ecuación. 139-140 Pp.

Rojas, V. (2009) Filosofía del Derecho, Oxford, segunda edición, Oxford: México. 206 Pp.

Searle, J. (2014) Creando el mundo social la estructura de la civilización humana, trad. de Juan Bostelmann, México: Paidós. 252-253 Pp.

Revistas.

Arias Marín, J. Alan, y Sánchez Solano, Úrsula. (2008) “Comentario bibliográfico de la obra Los derechos humanos como política e idolatría”, Derechos humanos México, México: núm. 9. 187-201 pp.

Haba, E. (2002) "Metodología realista-crítica y ética del razonamiento judicial: (realismo jurídico como alternativa práctica al discurso normativista de los jueces)”. Alicante: Doxa. N. 25. 503-531 pp.

Tesis y jurisprudencia

Tesis I.2o.A.E.32 A, Semanario Judicial de la Federación y su Gaceta, Décima Época, t. IV, Mayo de 2016, p. 2740.

Tesis P./J. 20/2014, Semanario Judicial de la Federación y su Gaceta, Décima Época, t. I, Abril de 2014, p. 202.

Tesis 1a. CXLVIII/2014, Semanario Judicial de la Federación y su Gaceta, Décima Época, t. I, Abril de 2014, p. 801.

Tesis 1a. CCCXVII/2014, Semanario Judicial de la Federación y su Gaceta, Décima Época, t. I, Septiembre de 2014, p. 574. 
Tesis 2a. XCIII/2016, Semanario Judicial de la Federación y su Gaceta, Décima Época, t. I, Septiembre de 2016, p. 834.

Tesis 2a. CIX/2014, Semanario Judicial de la Federación y su Gaceta, Décima Época, t. I, Noviembre de 2014, p.1190

Tesis 2a. XCIII/2016, Semanario Judicial de la Federación y su Gaceta, Décima Época, t. I, Septiembre de 2016, p. 834.

Tesis I.4o.A.12 K, Semanario Judicial de la Federación y su Gaceta, Décima Época, l. XVII, Febrero de 2013, p. 1345. Legales.

Ley Disciplina Financiera de las Entidades Federativas y los Municipios. Diario Oficial de la Federación. México. 27 de abril de 2016.

Presupuesto de Egresos de la Federación para el Ejercicio Fiscal 201\%. Diario Oficial de la Federación. México. 30 de noviembre de 2016.

Presupuesto de Egresos de la Federación para el Ejercicio Fiscal 2016, Diario Oficial de la Federación. México. 27 de noviembre de 2015.

Electrónicas.

Bolívar, L. (1996) “Derechos Económicos Sociales y Culturales: derribar mitos, enfrentar retos, tender puentes. Estudios Básicos de Derechos Humanos. [En Línea]. Disponible en: http://www.corteidh.or.cr/tablas/a12003.pdf

Casar, M. (2015). Anatomía de la Corrupción. México: CIDE [En línea]. Disponible en:http://imco.org.mx/wpcontent/uploads/2015/05/2015_Libro_completo_Anatomia_corrupcion.pdf

CONEVAL. (2014) “Índice de Medición de la Pobreza” [En Línea]. Disponible en:

http://www.coneval.org.mx/Medicion/MP/Paginas/Pobreza_2014.aspx

OECD. (2016). Panorama de la educación 2015: Indicadores de la OCDE. Madrid: Fundación Santillana. [En Línea]. Disponible en: http://dx.doi.org/10.1787/eag-2015-es 